\title{
Regulation of Biochemical Plant Growth Regulators at the U.S. Environmental Protection Agency
}

\author{
Sheryl K. Reilly, ${ }^{1}$ Leslie K. Lake, ${ }^{2}$ Warren E. Shafer, ${ }^{3}$ and Russell S. Jones ${ }^{1}$
}

AdDitional INDEX wORDs. plant hormones, biochemical pesticides, biopesticides, agrochemical product registration, Biopesticides and Pollution Prevention Division, BPPD

Summary. Plant growth regulators (PGRs) are often used in crop production for specific niche market needs. PGRs are frequently viewed as secondary business opportunities by the private sector, especially when compared to herbicide, insecticide, and/or fungicide markets. Nonetheless, PGRs are regulated by the U.S. Environmental Protection Agency (USEPA), and the additional cost of regulatory compliance as part of commercial development is significant. Of the two broad classes of pesticides regulated by the USEPA, conventional chemicals and biological pesticides (or biopesticides), many PGRs belong to the biopesticide class, specifically the biochemical category. Because of USEPA's responsibility to assure that any pesticide used in commerce will not result in unreasonable adverse effects to humans or the environment, specific data requirements have been established for product registration. Registrants must address each requirement, either by submitting relevant data or a request to waive the requirement, prior to receiving a federal registration. For biochemical PGRs, the acceptability of data or waiver requests, as well as any proposed label uses, are reviewed by the Biopesticides and Pollution Prevention Division (BPPD). The BPPD was formed in 1994 to facilitate the development of biopesticide products. Given the time and expense associated with PGR product development and commercialization, registrants should work closely with the USEPA and other stakeholders to help ensure successful product development.

lant growth regulators are important production tools for a
variety of horticultural and agronomic crops (for examples, see
Cutler and Schneider, 1990; Meister Publishing, 2000). While their effects, particularly in an overdose situation, can be quite profound, PGRs are typically viewed as products that can be used to subtly manipulate a given plant growth process (e.g., flowering, vegetative shoot elongation, fruit abscission). The value they create, particularly in the horticultural crop arena, can be quite significant and multidimensional [e.g., the use of naphthaleneacetic acid on apples (Malus domestica) as a thinning agent early in the season, then as a preharvest drop control agent late in the season]. Current market trends, wherein demand for premium crop quality is growing at the same time input costs are being squeezed, will likely continue the drive to develop new PGR products that can help growers effectively achieve this marketplace balance.

${ }^{1}$ Biochemical Pesticides Branch, Biopesticides and Pollution Prevention Division (7511C), U.S. Environmental Protection Agency, 1921 Jefferson Davis Highway, Room 910D, Crystal Mall 2, Arlington, VA 22202.

${ }^{2}$ Health and Food Technologies, Cargill, Inc., 15407 McGinty Road, Wayzata, MN 55391.

${ }^{3}$ Renessen LLC, Suite 300 South, 3000 Lakeside Drive, Bannockburn, IL 60015; corresponding author. 
The development and ultimate registration of a new PGR product involves an assessment of field performance (efficacy) as well as careful examination of its safety profile relative to humans and the environment. Data developed in support of registration are used to determine application rates, use instructions, precautionary information, worker protection requirements, and storage and disposal information-all of which are ultimately described on the product label. Prerequisites for the development of data to support labeled uses include an understanding of the physiological effects of the PGR on target plant species, as well as the testing protocols and data required by the USEPA to determine safety for the intended use(s).

There are several publications that provide background and historical perspective to the procedures used for federal registration of biopesticides. These include Conner et al. (1991), which provides a general overview of the registration process, as well as some specific guidance concerning data requirements. McClintock et al. (1994) reviews the policies and practices that the USEPA uses to evaluate the health effects of biochemical pesticides. For a historical perspective, Plimmer and Parry (1994) discuss some of the early impediments to securing regulatory approvals. Finally, Mendelsohn et al. (1995) provide insight into the effect of product formulation on data requirements - an important parameter for new (PGR) product development.

The aim of our paper is to provide a brief overview of the USEPA regulatory process governing a particular class of PGRs, namely those considered biochemicals.

\section{Regulatory background}

The USEPA is authorized to regulate the use, sale, and distribution of conventional chemical and biological pesticides to ensure their use does not cause unreasonable adverse effects to humans or the environment. USEPA's authority to regulate pesticides is derived primarily from three statutes: 1 ) the Federal Insecticide, Fungicide, and Rodenticide Act (FIFRA) as amended, 2 ) the Federal Food, Drug, and Cosmetic Act (FFDCA), and 3) the Food Quality Protection Act(FQPA) of 1996.

A pesticide is broadly defined as any substance or mixture of substances intended for preventing, destroying, repelling, or mitigating any pest, or intended for use as a plant regulator, defoliant, or desiccant [Title 40 Code of Federal Regulations (40 CFR), part 152.15 (Office of the Federal Register (OFR), 2000); FIFRA, section 2(v) (USEPA, 200la)]. Pesticides, including PGRs, are subject to FFDCA [Title 21 U.S. Code, section 321 et seq. (U.S. Food and Drug Administration (USFDA), 1999)] if their use results in pesticide chemical residues in or on food commodities or animal feed. Under FFDCA, the USEPA is required to establish tolerances for pesticide residues on food or feed crops. Exemptions from the requirement of a tolerance may be granted by the USEPA when the risk to humans or the environment is low (due to lack of dietary exposure and/or low toxicity). In 1996, FQPA amended FIFRA and FFDCA to consider aggregate exposure to pesticides, including dietary and nondietary exposures, the cumulative effects of chemicals with common toxic mechanisms, and the variability or sensitivity of major population subgroups, including infants and children. All pesticides, including biochemical PGRs, are subject to the requirements of the amended act. For an overview of pesticides and human health risk assessment, see Whitford, et al. (1999). Also, Purdue University has a website that lists numerous publications that provide a wide variety of information regarding pesticides (Purdue University, 2001).

\section{Biopesticides and Pollution Prevention Division (BPPD)}

In response to increasingly stringent regulations calling for greater proof of the environmental safety of new agrochemicals, industry and academic scientists have poured significant resources into the development of new biopesticide products which pose a reduced risk to humans or the environment. However, the commercialization of some of these products may have been deterred by the economics surrounding their development for a limited, or niche, market due to the significant costs of registering these materials in the U.S. In 1994, the USEPA formed BPPD, a new division in the Office of Pesticide Programs (OPP), to help facilitate the registration (and reregistration) of biological products (Andersen et al., 1996).

Before the formation of BPPD, biopesticides were registered in OPP's Registration Division and reregistered in the Special Review and Reregistration
Division, in much the same manner as a conventional chemical pesticide. Both of these conventional pesticide regulatory divisions depend upon other science divisions for risk assessment and risk/benefit analysis (Health Effects Division, Ecological Fate and Effects Division, and Biological and Economic Analysis Division). BPPD is a multidisciplinary division with a staff consisting of both risk managers and risk assessors that handles virtually all aspects of the registration and reregistration processes for biopesticides in the U.S. This one-stop shopping design facilitates the registration process for biopesticides. In addition, OPP's Pesticide Environmental Stewardship Program staff in BPPD works closely with Interregional Research Project Number Four(IR-4), U.S. Department of Agriculture (USDA), USFDA and pesticide user groups to assist in the development of integrated pest management (IPM) programs. PGRs and other biopesticides play a significant role in this process; it is in this liaison role that the division derives the pollution prevention part of its name. For a comprehensive listing of all active ingredients that BPPD manages, see USEPA (2001b).

\section{Classification of biopesticides}

Regulated biological pesticides, or biopesticides, are divided into three categories: 1) microbial pesticides, 2) plant incorporated protectants (plants that produce their own pesticidal substances, having been genetically transformed to do so. USEPA regulates only the pesticide and the genetic machinery needed for the plant to produce it.), and 3) biochemical pesticides. Based on their mode of action, biochemical pesticides are further classified into functional classes, including semiochemicals (pheromones), natural insect growth regulators (IGRs), and natural PGRs (McClintock, et al., 1994). Several important PGRs (e.g., gibberellins, indole-3-butyric acid, ethylene) are included in the biochemical pesticide category. By definition, this means they possess a nontoxic mode of action and are naturally occurring (or structurally similar and functionally identical to a naturally occurring substance).

Biochemical pesticides are distinguished from conventional chemical pesticides by their natural occurrence and nontoxic mode of action to the 
target pest species. Examples of chemical classes exhibiting nontoxic modes of action include: insect lures (e.g., pheromones), attractants and repellents, irritants, suffocants (such as oils for insect control), and in terms of PGRs, changes in plant growth and development. Additional distinguishing characteristics for biochemical pesticides are their low use or application rates and a narrow range of target species (for PGRs, this equates to demonstrated beneficial effects on a limited range of target crops). For example, gibberellic acid is commercially registered and used in California for thinning seedless table grapes (Vitis vinfera). Use concentrations are in the low parts-per-million range, and crop responsiveness can vary significantly among cultivars (e.g., 'Thompson Seedless,' 'Perlette').

It should be noted that a synthetic active ingredient can be classified as a biochemical pesticide if it is structurally similar and functionally identical to a naturally occurring active ingredient. The best example of this is a pheromone, which is difficult to harvest in sufficientquantities from natural sources, but can be synthesized and produced in economically feasible quantities. Synthetic compounds can thus be classified as biochemical pesticides. Likewise, it is important to note that natural is not always synonymous with a nontoxic mode of action. For example, pyrethrins, heavy metals, arsenic, and antibiotics from microorganisms are naturally occurring, but can be highly toxic to a target pest. Therefore, per its charter, $\mathrm{BPPD}$ is not responsible for the regulatory review of all naturally occurring pesticidal active ingredients.

\section{Registration requirements}

Procedures for the registration of pesticides are set forth primarily in 40 CFR, parts 152 and 158 (OFR, 2000). Registrants of pesticides are required to satisfy a generic set of data requirements, as well as product-specific requirements appropriate to the end-use formulation. While the generation of relevant, scientifically valid data is often the most straightforward approach to addressing each requirement, the USEPA will consider data waiver requests, as long as they are based on a reasonable and defensible rationale [see 40 CFR, part 158.45 (OFR, 2000)]. Waiver requests, which are essentially white paper arguments as to why a given study is not required, are considered case by case. To help navigate through the regulatory process, registrants are definitely encouraged to meet with the BPPD for a preregistration conference to determine the best strategies for addressing data requirements for a new active ingredient and its intended use(s).

Due to their niche market applications and unique properties, the USEPA developed registration mechanisms and a modified set of data requirements to facilitate the review and approval of biopesticides, including biochemical PGRs. In general, data requirements for biopesticides include 1) product chemistry (e.g., product identity, analytical methods, manufacturing processes), 2) mammalian toxicology (to assess potential human health effects), and 3) nontarget organism effects. The data requirements are summarized in 40 CFR, part 158.690 (OFR, 2000); see also USEPA (2001c), which includes a crosswalk between the old guideline numbering system (USEPA, 1982, 1989) and the new Office of Prevention, Pesticides, and Toxic Substances (OPPTS) series of harmonized guidelines (USEPA, 1995a, 1995b, 1996, 1998a, 1998b). As with all pesticides, the USEPA has waived the requirement for registrants to submit product performance (efficacy) data unless the claims made are for microorganisms that pose a threat to human health, vertebrates (such as rodents, birds, bats, skunks) and insects that can directly or indirectly transmit diseases to humans. However, the USEPA does reserve the right to ask for efficacy data, and BPPD does ask for this case by case, when the proposed use(s) is (are) questionable, to ensure that biopesticides are not dismissed as a whole by consumers who may have found that a product that does not work in the manner described on the label.

In terms of product chemistry, the data requirements are focused on providing information to the USEPA about how the active ingredient is manufactured and ultimately formulated. Examples of requirements in this category include 1 ) information pertaining to the starting materials used to make the active ingredient, 2) a description of the manufacturing method used to produce the active ingredient, 3 ) a discussion regarding the formation of any unintentional ingredients or impurities, including their possible toxicological significance, 4) the determination of certified limits, and 5) an analysis of various physical/chemical properties (e.g., color, odor, melting point, solubility). The physical/chemical data requirements are found at $40 \mathrm{CFR}$, part 158.690 (OFR, 2000).

Mammalian toxicology data requirements for biochemical pesticides were developed by the USEPA together with experts from industry and academia as a "tiered" approach. For biochemical PGRs intended for use on terrestrial food crops, Tier I studies that are generally required include 1) acute toxicity (oral, dermal, and inhalation routes of administration),2) primary eye and dermal irritation, 3) dermal sensitization, 4) genotoxicity, 5) immunotoxicity, 6) 90-d feeding, 7) developmental toxicity, and 8 ) reporting of hypersensitivity incidents. These requirements are summarized in table (c) of $40 \mathrm{CFR}$, part 158.690 (OFR, 2000). If any of the Tier I studies or information from other sources (National Toxicology Program cancer studies, for example) triggers the need for additional testing, then BPPD can request that the registrant submit higher Tier II or Tier III studies, based upon the potential risks.

Studies that are required by the USEPA to assess effects of a biochemical on nontarget organisms and the environment are provided in table (d) of 40 CFR, part 158.690 (OFR, 2000). Tier I data requirements include 1 ) avian acute oral toxicity, 2) avian dietary toxicity, 3) freshwater fish testing, 4) freshwater aquatic invertebrate testing, 5) nontarget plant studies, and 6) nontarget insect testing. As with mammalian toxicology data requirements, the requirement for Tier II and Tier III study data for nontarget organisms (and possibly environmental fate) is contingent upon the outcomes from the Tier I studies.

It should be noted that procedures and requirements for experimental use permits (EUPs) are provided in $40 \mathrm{CFR}$, part 172 (OFR, 2000). BPPD issues EUPs for the testing of a new active ingredient (or a new use for an existing active ingredient) on more than 10 acres (4.0 ha) per pest. EUPs are important in the commercial development of PGRs because data may be generated to evaluate the feasibility of pursuing a registration, and the data can subsequently be used for future registration purposes. However, if the holder of the EUP desires to sell a crop tested under the experimental program, a temporary tolerance (or exemption from a tolerance) must be established, unless a per- 
manent tolerance (or exemption) already exists for the active ingredient. If there is no tolerance or exemption for the active ingredient, the permit holder must petition the USEPA for a temporary tolerance (or exemption) that will extend over the time period needed for the EUP. Procedures for filing petitions for pesticide food tolerances and exemptions are described and listed in 40 CFR, part 177 (OFR, 2000), and already established tolerances and exemptions are located in $40 \mathrm{CFR}$, part 180 (OFR, 2000).

For further information regarding the registration of biopesticides, including guidelines for performing the required studies for biochemical PGRs, see OPP's website (USEPA, 2001d). BPPD personnel are also available for consultation concerning the data required for registering biopesticide products.

\section{Conclusions}

PGRs are important tools that are routinely used in the production of a variety of horticultural and agronomic crops. From flower and fruit thinning, to height control, to the manipulation of pigmentation or senescence processes, PGRs help create significant economic and aesthetic value. While PGRs are often considered niche market products, numerous growers, distributors, processors, and ultimately, the consumer, all benefit from the positive effects they impart. Increasing pressure to minimize crop inputs while maximizing harvest endpoints, such as outlined above, and to reduce the use of conventional pesticides by developing IPM programs that incorporate their use, will undoubtedly continue to drive the future development of new PGR products.

To maximize the likelihood of timely success, the process of registering a PGR in the U.S. requires a collaborative effort between the registrant, the USEPA, IR-4, USDA, USFDA, states, tribes and regions, and other resource groups (e.g., growers, university researchers, extension specialists, industry trade groups). These stakeholders should work together to develop an understanding of the safety, performance, benefits, and environmental impact of new agricultural production tools such as PGRs. It is important that all involved parties understand the regulatory construct under which these products are regulated and that they develop an understanding of, and appreciation for, the data required for product registration.

\section{Literature cited}

Andersen, J., A. Leslie, S. Matten, and R. Kumar. 1996. The Environmental Protection Agency's programs to encourage the use of safer pesticides. Weed Technol. 10:966-968.

Conner, Jr., J.D., L.S. Ebner, S.W. Landfair, C.A. O'Connor, III, K.W. Weinstein, and A.P. Jovanovich. 1991. Pesticide regulation handbook. $3^{\text {rd }}$ ed. Executive Enterprises Publ., New York.

Cutler, H.G. and B.A. Schneider. 1990. Plant growth regulator handbook of the Plant Growth Regulator Society of America. $3^{\text {rd }}$ ed. Plant Growth Regulator Soc. Amer., LaGrange, Ga.

McClintock, J.T., J.L. Kough, and R.D. Sjoblad. 1994. Regulatory oversight of biochemical pesticides by the U.S. Environmental Protection Agency: Health effects considerations. Regulat. Toxicol.Pharmacol. 19:115-124.

Meister Publishing. 2000. Farm chemicals handbook. vol. 86. Meister Publ., Willoughby, Ohio.

Mendelsohn, M.L., T.C. Ellwanger, R. I. Rose, J.L. Kough, and P.O. Hutton. 1995. Registration of biologicals: How product formulations affect data requirements, $\mathrm{p}$. 20-26, In: F.R. Hall and J.W. Barry (eds.). Biorational pest control agents: Formulation and delivery. Amer. Chem. Soc. Symp. Ser. 595 .

Office of the Federal Register. 2000. Code of federal regulations, protection of the environment, title 40, parts 150-189. Natl. Archives and Records Admin., Wash., D.C.

Plimmer, J.R. and R.M. Parry, Jr. 1994. Registration of biopesticides, p. 474-489. In: P.A. Hedin, J.J. Menn, and R.M. Hollingworth (eds.). Natural and engineered pest management agents. Amer. Chem. Soc. Symp. Ser. 551.

Purdue University. 2001. Purdue University pesticide programs. 17 Oct. 2001. <http://www.btny.purdue.edu/PPP/>.

U.S. Environmental Protection Agency. 1982. Pesticide assessment guidelines, subdivision $M$, biorational pesticides. U.S. EPA, Office of Pesticides and Toxic Substances, Wash., D.C.

U.S. Environmental Protection Agency. 1989. Subdivision M of the pesticide testing guidelines, microbial and biochemical pest control agents. 1989. U.S. EPA, Office of Pesticides and Toxic Substances, Wash., D.C.
U.S. Environmental Protection Agency. 1995a. Office of Prevention, Pesticides, and Toxic Substances (OPPTS) harmonized test guidelines: Biochemicals test guidelines. OPPTS Series 880. U.S. EPA, Wash., D.C.

U.S. Environmental Protection Agency. 1995b. Office of Prevention, Pesticides, and Toxic Substances (OPPTS) harmonized test guidelines: Microbial pesticide test guidelines, OPPTS Series 885. U.S. EPA, Wash., D.C.

U.S. Environmental Protection Agency. 1996. Office of Prevention, Pesticides, and Toxic Substances (OPPTS) harmonized test guidelines: Product properties, OPPTS Series 830. U.S. EPA, Wash., D.C.

U.S. Environmental Protection Agency. 1998a. Office of Prevention, Pesticides, and Toxic Substances (OPPTS) harmonized test guidelines: Health effects, OPPTS Series 870. U.S. EPA, Wash., D.C.

U.S. Environmental Protection Agency. 1998b. Office of Prevention, Pesticides, and Toxic Substances (OPPTS) harmonized test guidelines: Product performance test guidelines, OPPTS Series 810. U.S. EPA, Wash., D.C.

U.S. Environmental Protection Agency. 200la. Federal insecticide, fungicide, and rodenticide act (FIFRA). 31 Oct. 2001. <http://www.epa.gov/pesticides/ fifra.htm>.

U.S. Environmental Protection Agency. 2001b. Biopesticides-All active ingredients. 17 Oct. 2001 < <ttp:// www.epa.gov/pesticides/biopesticides/ ai/all_ais.htm>.

U.S. Environmental Protection Agency. 2001c. Biochemical pesticide test guidelines. 17 Oct. 2001. <http:// www.epa.gov/pesticides/biopesticides/ guidelines/biochem2.htm>.

U.S. Environmental Protection Agency. 2001d. Biopesticides. 15 Oct. 2001. <http://www.epa.gov/pesticides/ biopesticides $>$.

U.S. Food and Drug Administration. 1999. The Federal Food, Drug and Cosmetic Act (FFDCA). 31 Oct. 2001. <http:// www.fda.gov/cvm/index/ffdc_act/ ffdcatoc.html $>$.

Whitford, F., J. Kronenberg, C. Lunchick, J. Driver, R. Tomerlin, J. Wolt, H. Spencer, C. Winter, and G. Whitmyre. 1999. Pesticides and human health risk assessmentPolicies, processes, and procedures (PPP48). Purdue Pesticide Programs, Purdue Univ. Coop. Ext. Serv., West Lafayette, Ind. 